\title{
The emerging phenotype of long-term survivors with infantile Pompe disease
}

\author{
Sean N. Prater, MRes' ${ }^{1}$, Suhrad G. Banugaria, MBBS'1, Stephanie M. DeArmey, PA-C', \\ Eleanor G. Botha, CGC², Erin M. Stege, CPNP³, Laura E. Case, DPT ${ }^{4}$, Harrison N. Jones, PhD ${ }^{5}$, \\ Chanika Phornphutkul, MD', Raymond Y. Wang, MD ${ }^{7,8}$, Sarah P. Young, PhD ${ }^{1}$ \\ and Priya S. Kishnani, MD
}

\begin{abstract}
Purpose: Enzyme replacement therapy with alglucosidase alfa for infantile Pompe disease has improved survival creating new management challenges. We describe an emerging phenotype in a retrospective review of long-term survivors.

Methods: Inclusion criteria included ventilator-free status and age $\leq 6$ months at treatment initiation, and survival to age $\geq 5$ years. Clinical outcome measures included invasive ventilator-free survival and parameters for cardiac, pulmonary, musculoskeletal, gross motor and ambulatory status; growth; speech, hearing, and swallowing; and gastrointestinal and nutritional status.
\end{abstract}

Results: Eleven of 17 patients met study criteria. All were cross-reactive immunologic material-positive, alive, and invasive ventilatorfree at most recent assessment, with a median age of 8.0 years (range: 5.4-12.0 years). All had marked improvements in cardiac parameters. Commonly present were gross motor weakness, motor speech

\section{INTRODUCTION}

Pompe disease (OMIM 232300) is a lysosomal storage disorder caused by deficiency of acid $\alpha$-glucosidase (GAA; OMIM 606800), ${ }^{1}$ resulting in rapid accumulation of lysosomal glycogen in skeletal, cardiac, bulbar, and smooth muscle. ${ }^{1-4}$ Classical infantile Pompe disease (IPD) is characterized by cardiomyopathy, hypotonia, respiratory insufficiency, and death secondary to cardiorespiratory failure by the age of 2 years. ${ }^{1,5,6}$ Treatment for IPD by enzyme replacement therapy (ERT) with alglucosidase alfa (Myozyme, rhGAA; Genzyme, Cambridge, MA) has been available since 2006 and has led to improvements in survival rates and clinical outcomes. ${ }^{5,7}$ Infants in the pivotal trial of rhGAA had variable clinical outcomes, and this variability became more apparent over time, with $\sim 50 \%$ of the patients being dependent on invasive ventilation when followed up to the age of 41.5 months. ${ }^{8}$ The marked variability in clinical outcomes with ERT is believed to result from multiple factors, including age at initiation of ERT, extent of disease-related pathology at baseline, underlying genotype, cross-reactive immunologic material deficits, sensorineural and/or conductive hearing loss, osteopenia, gastroesophageal reflux, and dysphagia with aspiration risk. Seven of 11 patients were independently ambulatory and four required the use of assistive ambulatory devices. All long-term survivors had low or undetectable anti-alglucosidase alfa antibody titers.

Conclusion: Long-term survivors exhibited sustained improvements in cardiac parameters and gross motor function. Residual muscle weakness, hearing loss, risk for arrhythmias, hypernasal speech, dysphagia with risk for aspiration, and osteopenia were commonly observed findings.

Genet Med 2012:14(9):800-810

Key Words: acid maltase deficiency, enzyme replacement therapy, glycogen storage disease type II, neuromuscular diseases, Pompe disease

(CRIM) status, differences in muscle fiber type (i.e., type I versus type II) distribution, defective autophagy, and high sustained antibody titers. ${ }^{7-13}$

Although more than a decade has passed since the first clinical trials, there is still a paucity of data on long-term outcomes, with follow-up data available only up to age 41.5 months at the time of this report. ${ }^{8}$ Our study sought to characterize the longterm clinical phenotype of IPD and the emerging clinical challenges associated with chronic ERT administration.

\section{MATERIALS AND METHODS}

Medical records relating to IPD from July 1999 to June 2011 were reviewed. Inclusion criteria were (i) onset of symptoms by $\leq 6$ months per the corrected gestational age (i.e., the patient's gestational age corrected to 40 weeks if born prior to 40 weeks of gestation), (ii) GAA activity in skin fibroblasts and/or muscle biopsy $<1 \%$ of the control mean value, (iii) the presence of cardiomyopathy (left-ventricular mass index (LVMI) values $>64 \mathrm{~g} / \mathrm{m}^{2}$ (ref. 14)) and absence of ventilatory support before

\footnotetext{
${ }^{1}$ Division of Medical Genetics, Department of Pediatrics, Duke University Medical Center, Durham, North Carolina, USA; ${ }^{2}$ Department of Human Genetics, Emory University School of Medicine, Atlanta, Georgia, USA; ${ }^{3}$ Children's Hospital \& Research Center Oakland, Oakland, California, USA; ${ }^{4}$ Department of Community and Family Medicine, Division of Physical Therapy, Duke University Medical Center, Durham, North Carolina, USA; ${ }^{5}$ Department of Surgery, Division of Speech Pathology \& Audiology, Duke University Medical Center, Durham, North Carolina, USA; ${ }^{6}$ Department of Pediatrics, Division of Pediatric Endocrinology and Metabolism, Rhode Island Hospital and Brown University, Providence, Rhode Island, USA; ${ }^{7}$ Division of Metabolic Disorders, Pediatric Subspecialty Faculty, CHOC Children's, Orange, California, USA; ${ }^{8}$ Department of Pediatrics, University of California-Irvine School of Medicine, Orange, California, USA. Correspondence: Priya S. Kishnani (kishn001@mc.duke.edu)
} 
the start of ERT (baseline), (iv) age at ERT initiation $\leq 6$ months by corrected gestational age, and (v) survival to age $\geq 5$ years at the most recent assessment (MRA).

For study outcome measures, baseline and follow-up data were derived from clinical and laboratory evaluations performed by health-care professionals. Parents/guardians of all the patients provided informed consent under protocols approved by the institutional review boards. All the patients received alglucosidase alfa biweekly by infusion at cumulative doses of $20-40 \mathrm{mg} /$ $\mathrm{kg} .{ }^{15}$ Data regarding the cumulative dose versus time were collected for all the patients.

\section{Outcomes}

Patient outcomes were determined through analysis of clinical and laboratory data. Invasive ventilator-free survival was the primary outcome measure in this study, per the original clinical trials of alglucosidase alfa in infants treated at $\leq 6$ months of age. ${ }^{7}$ Secondary clinical outcome measures included cardiac, pulmonary, musculoskeletal, gross motor, ambulatory, swallowing, gastrointestinal, and nutritional status; physical growth (i.e., height, weight, occipitofrontal circumference (OFC)); and speech and hearing functions. Laboratory-based secondary outcome measures included rhGAA antibody titers, urinary glucose tetrasaccharide (Glc4), serum creatine kinase (CK), serum CK-MB isoform (CK-MB), and serum aspartate aminotransferase (AST) and alanine aminotransferase (ALT).

Pulmonary status was assessed through the documented use of respiratory support or supplemental oxygen, and obstructive sleep apnea as diagnosed through sleep studies.

Cardiac status. LVMI $\left(\mathrm{g} / \mathrm{m}^{2}\right)$ was assessed using two-dimensional, M-mode, and Doppler echocardiography. Given the increased risk for arrhythmia in IPD, ${ }^{16}$ electrocardiogram (ECG) data were used to assess PR, QRS, QT, and QTc intervals and history of supraventricular tachycardia, premature ventricular contractions, Wolff-Parkinson-White syndrome, and earlier interventions for arrhythmia control (cardiac-related medications and/or radiofrequency ablation).

Musculoskeletal, gross motor, ambulation, and bone density assessments. Capacity for independent ambulation, and use of ankle-foot orthoses or assistive mobility devices (e.g., walker or wheelchair) at MRA were recorded. Gross motor function was assessed using age-equivalent scores and percentiles, collected through the administration of age-appropriate standardized clinical instruments ${ }^{17}$ by a physical therapist. Alberta Infant Motor Scale score ${ }^{18}$ at baseline and at 12 months after ERT, and the Gross Motor Function Measure score ${ }^{19}$ at MRA were used. Assessment of residual motor weakness (generalized hypotonia and selective weakness in neck flexors, ankle dorsiflexors, and hip extensors; myopathic facies; and ptosis) at was performed at MRA by clinical examination and retrospective chart review. Given that osteopenia is associated with increased fracture risk over time in patients with IPD who are on $\mathrm{ERT},{ }^{20}$ the $z$-score data obtained at the most recent dual-emission X-ray absorptiometry (DXA) analysis of the L1-L4 lumbar spine were reviewed.

Physical growth. Age- and gender-appropriate percentiles for height, weight, and OFC at the time of MRA were calculated and compared with individual trends for each patient. ${ }^{21,22}$

Speech and hearing. Abnormal nasal resonance (i.e., hypernasality), a common observation in IPD, ${ }^{4}$ was selected as an outcome measure at MRA. The use of unilateral or bilateral hearing aids and/or tympanostomy tubes/grommets at MRA was assessed, and the types of hearing deficit (sensorineural and/or conductive) were categorized.

Swallowing, gastrointestinal, and nutrition. The presence of dysphagia with aspiration on videofluoroscopic swallow examination, gastroesophageal reflux disease, and overall mode(s) of nutritional intake (i.e., oral, tube feeds, or both) at MRA was assessed.

Laboratory methods. GAA mutation, CRIM status, antirhGAA immunoglobulin $\mathrm{G}$ (IgG) antibody titers, and urinary Glc4 analyses were performed as previously described., ${ }^{7,12,23,24}$

Statistical methods. Standard descriptive statistics were used to describe demographic and clinical characteristics. Pearson product-moment correlation coefficients were calculated using GraphPad Prism 5 software (La Jolla, CA). Statistical significance was defined as $\alpha=0.05$ (two-tailed). Given the limited sample size and nonstandard distributions of values assessed, the data relating to group outcome variables are presented as medians and ranges unless otherwise indicated. Mean urinary Glc4 and serum values of CK, CK-MB, AST, and ALT for each patient were determined for different time bins $(0-3,3-6,6-12$, $12-18,18-24,24-36,36-48$, and $48-60$ months). The number of observations varied from 0 to 10 per patient per time bin. Median values and ranges of binned mean data were calculated ( $n=5-9$ for each time bin). Glc4 trends were not monitored for patients 1 and 11 and have been reported earlier for patients 2 through 6 up to 32 months of therapy. ${ }^{7,24}$ The Wilcoxon matchedpairs signed-rank test $(\alpha=0.05)$ was used to assess serum CK trends by comparing median, first, and last $\mathrm{CK}$ values obtained for each patient ( $n=10$; no data available for patient 5$)$.

\section{RESULTS}

A total of 17 patients with IPD (12 male, 5 female; 11 CRIMpositive, 6 CRIM-negative), of whom some had participated in the original clinical trials of rhGAA, ${ }^{7-9,12}$ comprised the source population for this study. None of the CRIM-negative patients met the inclusion criterion of survival at $\geq 5$ years of age. Baseline demographics and clinical characteristics of the $11(8 \mathrm{M}, 3 \mathrm{~F})$ long-term survivors who met the inclusion criteria are shown in Table 1. The data obtained from monitoring up to 41.5 months of age in patients 1-6 have been previously published ${ }^{7-9,12}$ and an extension of those data is presented 


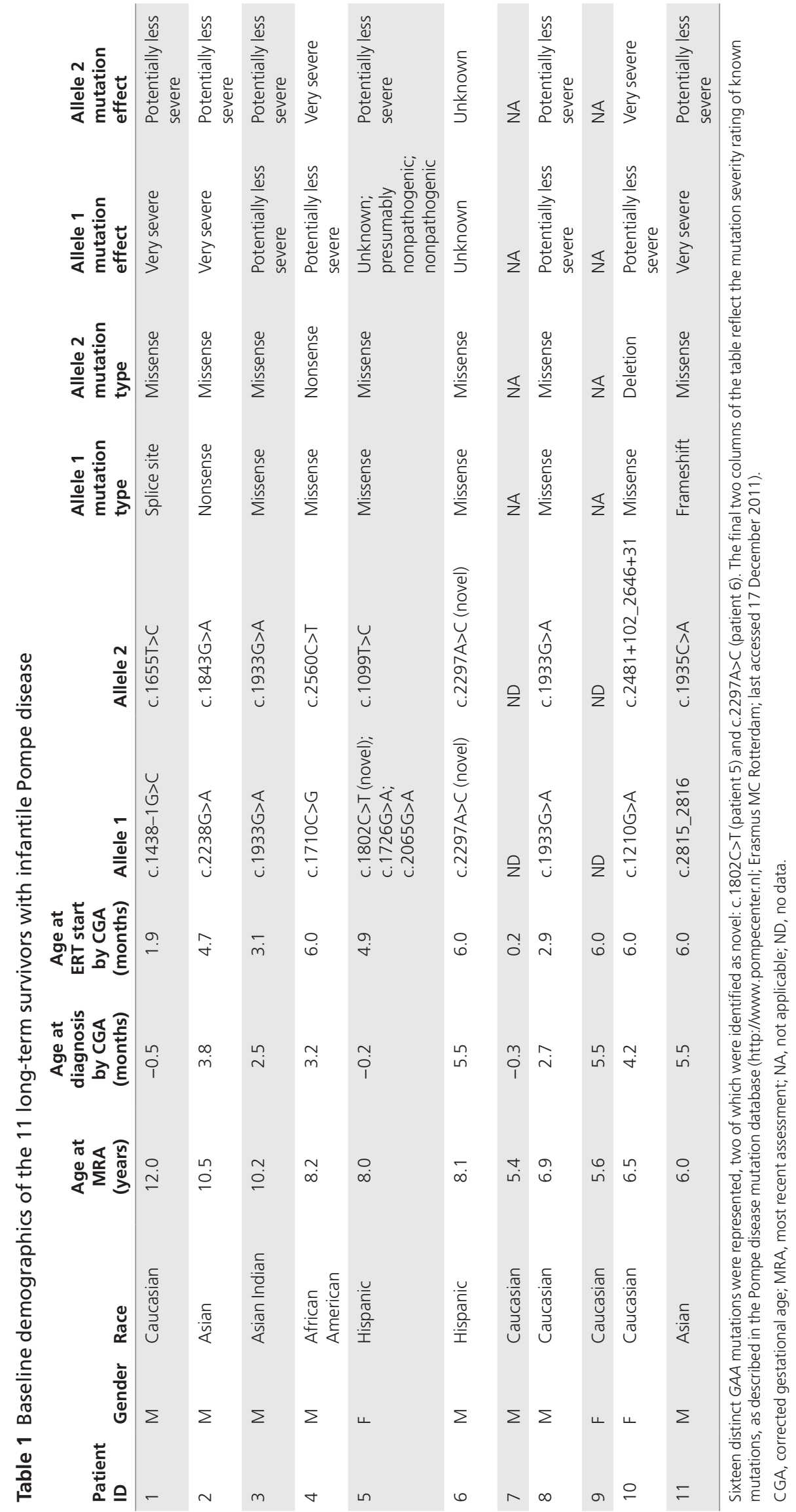


here. Data relating to patients 7 through 11 have not been published earlier. The cumulative biweekly dose of rhGAA dose $(\mathrm{mg} / \mathrm{kg})$ versus time (in months) for each of the 11 long-term survivors is shown in Supplementary Figure S1 online. The median age of a study patient at database lock was 8.0 years (5.4-12.0 years; $n=11)$; at diagnosis it was 3.2 months $(-0.5$ to 5.5 months; $n=11)$; and at ERT initiation it was 4.9 months ( 0.2 to 6.0 months; $n=11$ ). Table 1 lists GAA mutation data, available for 9 of the 11 patients. A total of 16 distinct GAA mutations were identified (11 missense, 2 nonsense, 1 frameshift, 1 deletion, and 1 splice-site mutation) and compared with those cited in published data in the Pompe disease mutation database (http://www.pompecenter.nl; Erasmus MC Rotterdam; last accessed 17 December 2011) ${ }^{23}$ and the Human Gene Mutation Database (http://www.hgmd.org; accessed 17 December 2011). Of the 16 mutations, 2 mutations were determined to be novel: c. $1802 \mathrm{C}>\mathrm{T}$ (patient 5) and c.2297A>C (patient 6). Known or putative mutation effects for published mutation(s) and a mutation severity rating adapted from the Erasmus MC Rotterdam human GAA mutation database are also shown (Table 1).

\section{Outcomes}

Pulmonary status. None of the 11 patients required ventilatory support of any type (i.e., invasive or noninvasive ventilation, including supplemental oxygen) during ERT, including at the time of MRA. At MRA, 2 of the 11 patients had mild obstructive sleep apnea as confirmed by a sleep polysomnogram (Table 2).

Cardiac status. The baseline cardiomegaly observed in all 11 long-term survivors resolved $\sim 5$ months after the start of ERT (Figure 1). The baseline median LVMI was $220 \mathrm{~g} / \mathrm{m}^{2}$ $\left(65-352 \mathrm{~g} / \mathrm{m}^{2} ; n=7\right.$; upper control limit for infants: $64 \mathrm{~g} / \mathrm{m}^{2}$ (ref. 14)); at all subsequent time points after 5 months, median LVMI values remained within control limits. The median duration of ERT treatment at MRA of LVMI was 69 months (28-110 months; $n=8$ ). Of the 11 patients, 5 (patients 2, 4, 5, 6 , and 11) had documented cardiac arrhythmia(s), presenting as supraventricular tachycardia (or, more precisely, as WolffParkinson-White syndrome ventricular pre-excitation syndrome), premature ventricular contractions, or as arrhythmia not otherwise specified. Patient 2 had an arrhythmia (supraventricular tachycardia) diagnosed before ERT initiation, and this was later treated with radioablation. The four other patients were diagnosed with arrhythmia while on ERT. Patients 4 and 11 were treated with $\beta$-blockers, whereas patients 5 and 6 had not received any treatment for arrhythmia. Long-term ECG data corresponding to PR, QRSD, QT, and QTc interval durations were within control ranges at different time points $(n=$ 10; data not shown).

Musculoskeletal, gross motor, ambulation, and bone density assessments. At MRA, 7 of the 11 patients were independently ambulatory without assistive devices, using independent ambulation as their primary means of mobility (patients 1-4, 7, 8, 10). Four patients (patients 5, 6, 9, and 11) required walkers on either a full-time or part-time basis: three patients were able to walk only with the full-time use of walkers (patients 6, 9, and 11), and two of these used walkers with pelvic supports (patients 6 and 9); patient 5, who had been independently ambulatory without assistive devices when younger, was still able to take some independent steps without an assistive device, but required part-time use of a walker at MRA. Ten of the patients wore ankle-foot orthoses. Median (range) Alberta Infant Motor Scale scores at baseline and at 12 months corresponded to the 1st (1st to 6th percentile; $n=$ 6 ) and 33 rd percentiles ( 1 st to 83 rd percentile; $n=6$ ), respectively. The median (range) age-adjusted Gross Motor Function Measure score at MRA corresponded to the 89th percentile (38th to 97th percentile; $n=7$ ). Overall, residual weakness was prevalent at MRA and included generalized hypotonia/weakness (10 of 11), neck flexor weakness (10 of 11), dorsiflexor weakness ( 9 of 11), hip extensor weakness ( 9 of 11), myopathic facies (11 of 11), and ptosis (5 of 11). DXA $z$-score data from the L1-L4 lumbar spine indicated that 6 of the 11 patients had osteopenia, defined as a DXA $z$-score greater than one s.d. below the age-adjusted mean (i.e., $z$-score $<-1.0$ ). Of the six patients with osteopenia, four had mild osteopenia $(-2.0<$ $z$-score $<1.0)$, and two had severe osteopenia $(z$-score $\leq-2.0)$ (Table 2). The range of DXA $z$-scores at MRA was -2.73 to 0.72 (median $z$-score: $-0.35 ; n=11$ ) and the median (range) duration on ERT at the most recent DXA assessment was 56 months (17-83 months; $n=11)$.

Physical growth. At MRA, all 11 patients had followed their typical curves for height, weight, and OFC, with values within the $3 \mathrm{rd}$ and 97 th percentiles for age and sex $(n=11),{ }^{21,22}$ except for patient 6 , whose OFC was below the 3rd percentile. Median (range) values for height, weight, and OFC at MRA corresponded to the 64th (9th to 94th percentile; $n=11$ ); 54th (10th to 97 th percentile; $n=11$ ); and 58th percentiles (1st to 97 th percentile; $n=8$ ), respectively.

Speech and hearing. At MRA, a majority of the patients had hypernasal speech (10 of 11) and a degree of hearing loss (9 of 11), including sensorineural hearing loss (9 of 11) and conductive hearing loss ( 4 of 11). Consequently, 7 of the 11 patients were using hearing aids and 3 of 10 patients had tympanostomy tubes/grommets.

Swallowing, gastrointestinal, and nutrition. At MRA, 7 of the 11 patients had an exclusively oral (PO) mode of nutrition intake; 4 patients used a combination of $\mathrm{PO}$ and gastrostomy tube feeds; 5 patients had dysphagia with aspiration on videofluoroscopic swallow examination; and gastroesophageal reflux disease was present in 3 patients.

Anti-rhGAA IgG antibodies. Serum anti-rhGAA IgG antibodies (Figure 2) developed in all 10 patients for whom data were available. At baseline, all 10 patients had been seronegative. The time to seroconversion ranged from 0.9 to 15 months (median: 


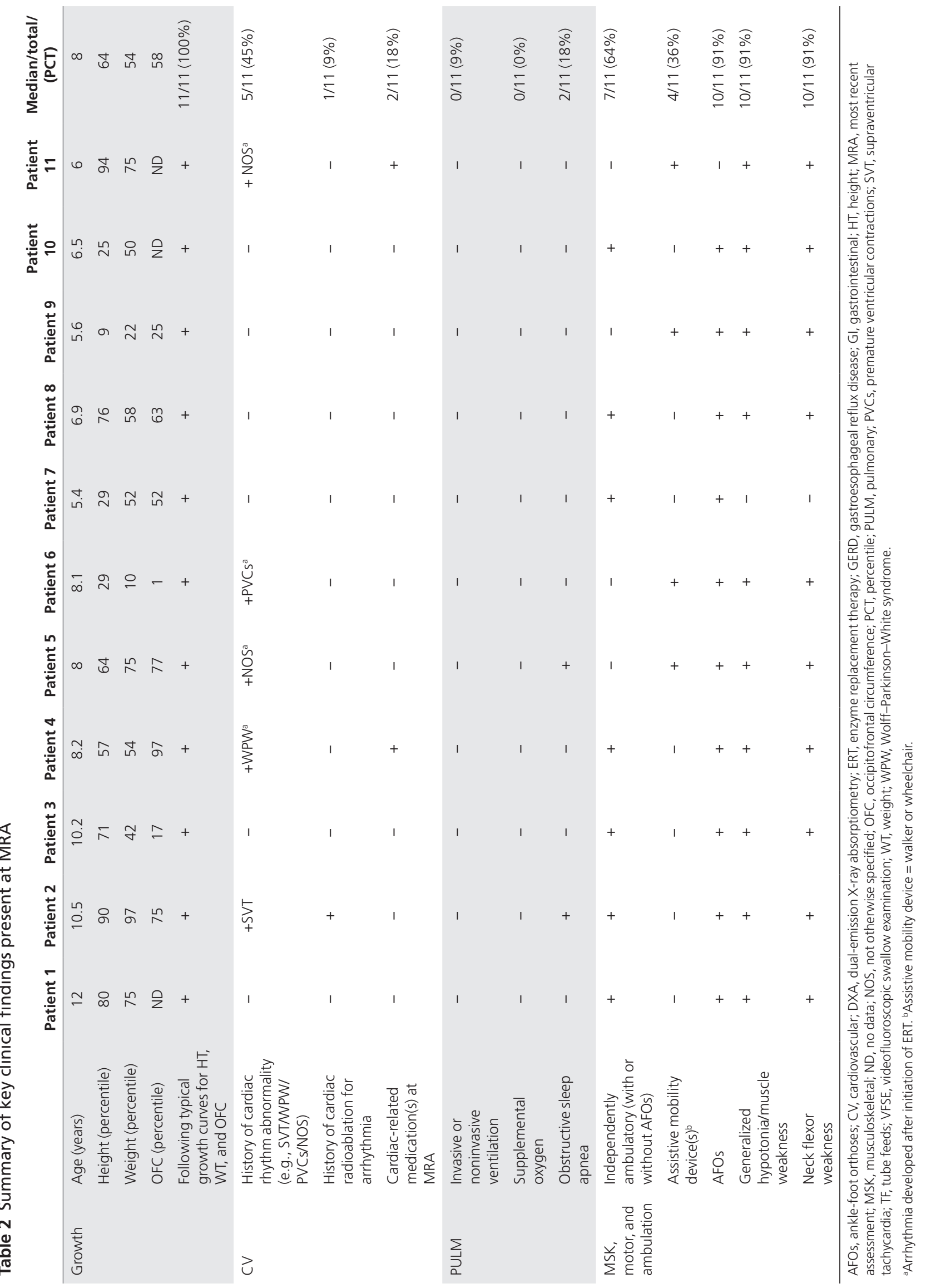

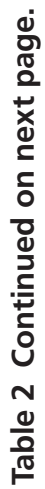




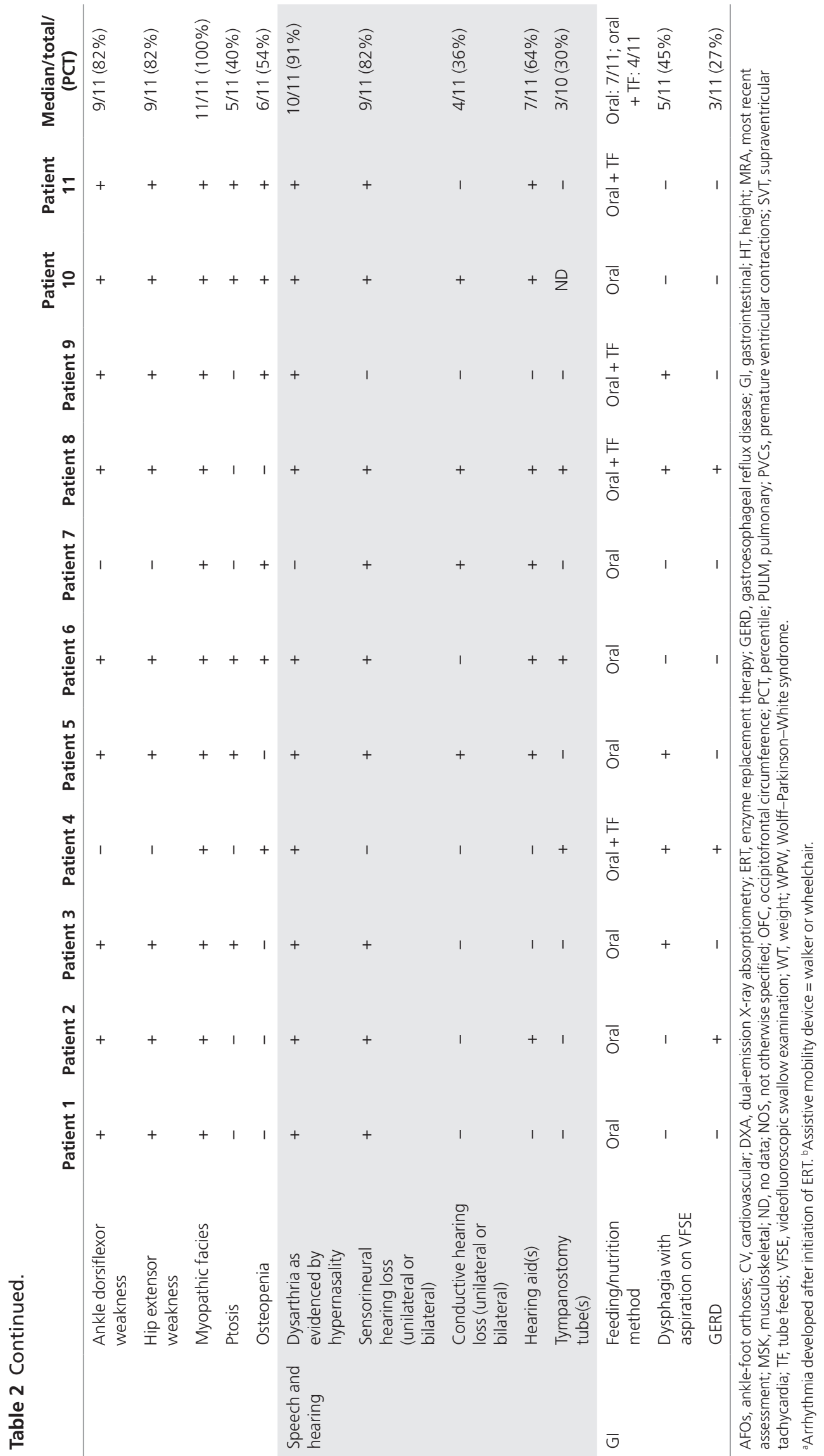




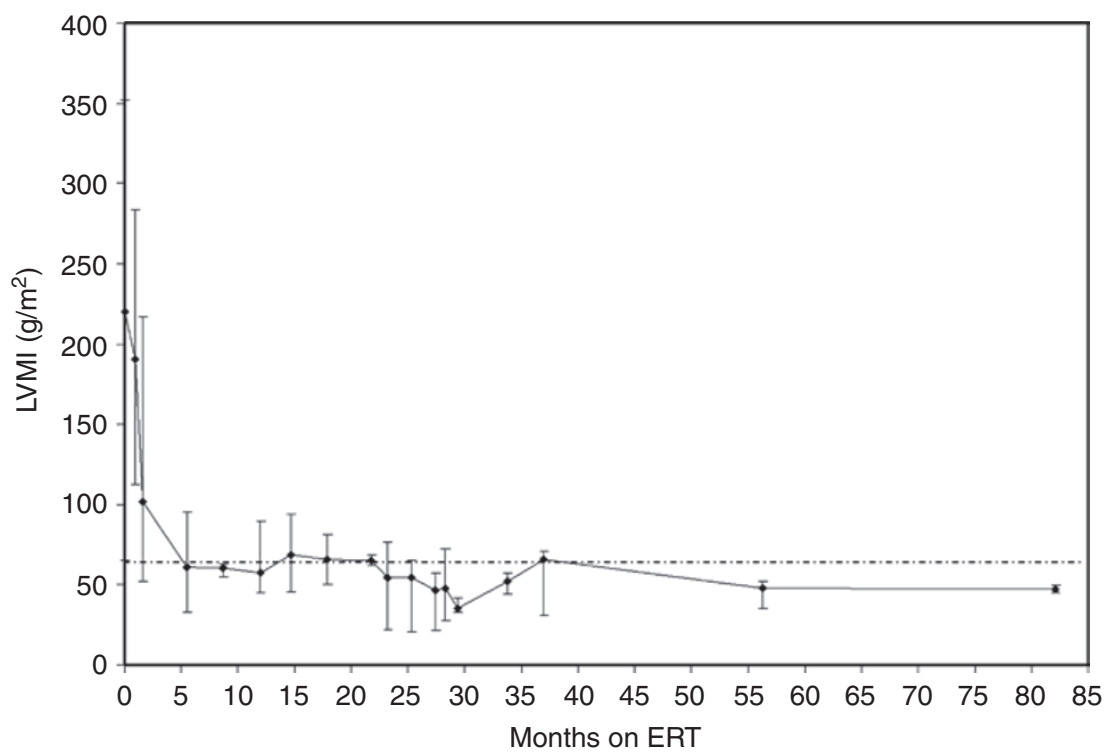

Figure 1 Median values and ranges for left-ventricular mass index (LVMI) versus months on ERT. The horizontal line indicates the upper limit of normal for LVMI $(\mathrm{g} / \mathrm{m})$ in healthy infants $\left(64 \mathrm{~g} / \mathrm{m}^{2}\right)(\mathrm{ref} .14)$. The median (range) LVMI value obtained at baseline was $220.3 \mathrm{~g} / \mathrm{m}^{2}\left(65.1-352.0 \mathrm{~g} / \mathrm{m}^{2} ; n=7\right)$. Median (range) LVMI values at 12 months after ERT initiation, 24 months after ERT initiation, and at MRA were $76.5 \mathrm{~g} / \mathrm{m}^{2}\left(45.0-89.8 \mathrm{~g} / \mathrm{m}^{2} ; n=9\right) ; 63.7 \mathrm{~g} / \mathrm{m}^{2}(26.6-76.6$ $\left.\mathrm{g} / \mathrm{m}^{2} ; n=7\right) ;$ and $47.4 \mathrm{~g} / \mathrm{m}^{2}\left(42.0-52.1 \mathrm{~g} / \mathrm{m}^{2} ; n=8\right)$, respectively. At MRA of LVMI, the median (range) duration on ERT was 69.3 months (28.1-109.9 months; $n=8)$. ERT, enzyme replacement therapy; MRA, most recent assessment.

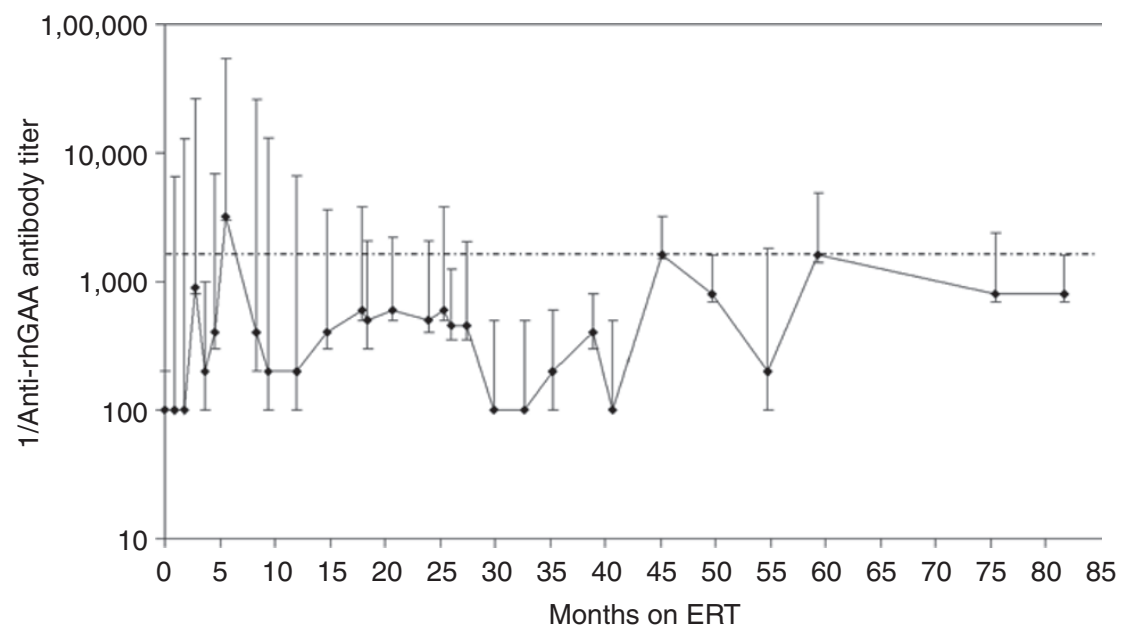

Figure 2 Medians and ranges for log 1/anti-rhGAA IgG antibody titers versus months on ERT. Longitudinal data were available for 10 of the 11 longterm survivors with IPD. The horizontal broken line represents a non-log-transformed titer of 1:1,600. As noted in an earlier study, 10 low-titer CRIM-positive patients had better clinical outcomes with ERT: non-log-transformed titers beyond 12 months after ERT initiation remained $\leq 1: 1,600$ (ref. 10). In our study of long-term survivors, median titers also remained consistently $\leq 1: 1,600$ beyond 12 months of ERT $(n=10)$. CRIM, cross-reactive immunologic material; ERT, enzyme replacement therapy; IgG, immunoglobulin G; IPD, infantile Pompe disease.

1.8 months), with most patients (8 of 10) developing antibodies by 2.8 months. Peak titers (median: 1:3,200, range: 1:200 to $1: 51,200 ; n=10$ ) developed in a wide range of 1.8 to 59 months (median: 5.5 months). At the time of MRA, all the patients had tolerized or had low titers (23-129 months ERT), ranging from 0 to $1: 1,600$.

Urinary Glc4. Glc4 trends were monitored in 9 of the 11 patients (Figure 3). Glc4 was elevated at baseline in seven patients for whom data were available, and these values decreased within 1 month of starting therapy. The lowest Glc4 values were observed during the first 2 years of ERT, after which increasing trends were observed in all the patients except patient 7. Median (range) urinary Glc4 values obtained at baseline, at 12 months, and at MRA (corresponding to 53-108 months on ERT) were $32 \mathrm{mmol} / \mathrm{mol}$ creatinine $(26-49 ; n=7), 14 \mathrm{mmol} / \mathrm{mol}$ creatinine $(7-36 ; n=8)$, and $52 \mathrm{mmol} / \mathrm{mol}$ creatinine (21-147; $n=9)$, respectively. 


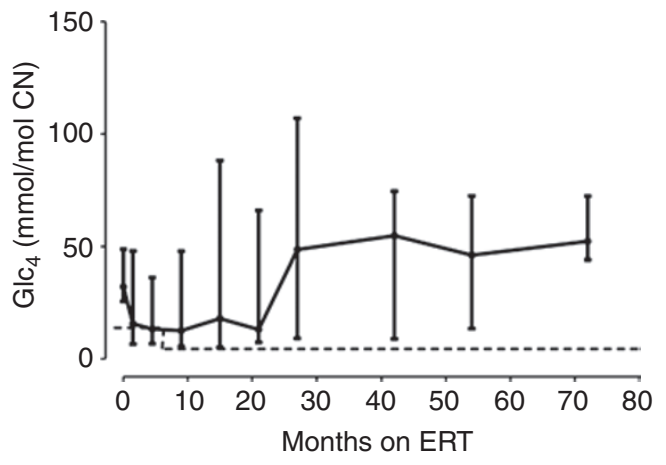

Figure 3 Trend in median urinary glucose tetrasaccharide (Glc4; in $\mathrm{mmol} / \mathrm{mol}$ creatinine (CN)) levels and ranges versus months on ERT. Urinary Glc4 is a biomarker for Pompe disease and is indicative of overall muscle glycogen burden. It is useful in monitoring treatment response. ${ }^{25}$ Data were available for 9 of the 11 patients. The broken line represents the upper limit of the range for age-matched controls (i.e., $14.0 \mathrm{mmol} / \mathrm{mol}$ $\mathrm{CN}$, age 6-12 months; $4.4 \mathrm{mmol} / \mathrm{mol} \mathrm{CN}$, age > 12 months). ERT, enzyme replacement therapy.

Serum CK, CK-MB, AST, and ALT. Baseline serum CK $(n=9)$, CK-MB $(n=10)$, AST $(n=9)$, and ALT $(n=9)$ levels were elevated or at the upper control limit in all the patients for whom data were available (Supplementary Table S1 online and Supplementary Figure S2a-d online). As with Glc4, the lowest median values for these serum biomarkers were observed during the first 2 years of treatment, after which they increased. However, whereas Glc4 median values decreased after 1 month on therapy, decreases in CK-MB, AST, and ALT occurred only after 4.5-10 months. CK median values did not decrease during the first 24 months of ERT and remained relatively constant. Moreover, comparisons of median CK values for the first and last CK evaluations, available for 10 of the 11 patients, showed no significant difference when assessed using the Wilcoxon matched-pairs signed-rank test $(P=0.22)$. The median (range) time on ERT treatment for which CK values were available was 54 months (21-72 months). Overall, CK, CK-MB, AST, and ALT values were persistently elevated during the course of the study in most of the patients for whom data were available. Additionally, all four serum biomarkers were significantly and positively correlated with one another and with urinary Glc4 values $(P<0.05)$.

\section{DISCUSSION}

In this study of long-term survivors with IPD, inclusion criteria were intentionally selected to mirror, as closely as possible, those used in the original pivotal trials of alglucosidase alfa $^{7-9,12}$ and to ensure that only the oldest patients were included. All the patients in this study had been, and continue to remain, free of invasive ventilation at MRA. LVMI trends in this cohort of patients, 5-12 years of age at MRA were reassuring; the LVMI normalization that occurred within 5 months after ERT initiation was sustained at MRA in all the patients. As demonstrated by findings from this and previous studies, ERT does not seem to be as effective in preventing the long-term development of arrhythmia. Patients with IPD have been shown to be at increased long-term risk for arrhythmia, ${ }^{25}$ with ECG findings often manifested as shortening of PR interval and/or prolongation of QRS or QTc intervals; patients with IPD are also at greater risk for Wolff-Parkinson-White syndrome and its attendant ECG changes. ${ }^{16,26,27}$ In this study, the development of an arrhythmia subsequent to initiation of ERT (seen in four of the five patients with a history of arrhythmia) further underscores the need for regular ECG assessment per published guidelines, particularly because some types of arrhythmia (e.g., tachydysrhythmias of Wolff-Parkinson-White syndrome as seen in patient 4) are considered potentially life-threatening and may result in sudden cardiac arrest. ${ }^{16,28}$ Arrhythmias may arise unpredictably in patients with IPD despite continued, regular administration of ERT. ${ }^{17,29,30}$ On the basis of previous findings using cardiac magnetic resonance imaging, it could be hypothesized that arrhythmias in long-term survivors may, at least in part, be related to myocardial fibrosis and/or ventricular remodeling associated with progressive long-term pathological changes in the heart. ${ }^{31}$ Progressive glycogen accumulation is known to occur in IPD in the myocardium as well as in the cardiac conduction system.

Although these long-term survivors continue to experience significant overall clinical benefit from ERT, closer examination clearly demonstrated that they have residual muscle weakness, and gross motor function below age-appropriate levels. Specific and notable areas of selective residual muscle weakness included the neck flexors, ankle dorsiflexors, hip extensors, abductors, and adductors, and the muscles of the face. Chronic residual weakness, especially when imbalanced across the joints of a growing child, may result in secondary musculoskeletal impairments. Hip-extensor weakness is often striking in these patients; when it occurs in conjunction with strong hip flexors, the resultant significant imbalance can become a deforming force. ${ }^{17}$ Profound weakness in ankle dorsiflexion can lead to hypoextensibility in the plantar flexors. ${ }^{17}$ Therefore, vigilance in assessment and management is recommended. ${ }^{17}$

Speech was also commonly affected in this group of survivors. Speech hypernasality, sound errors, and dysphonia were present in a majority of the patients at MRA. Structural defects did not appear to account for these speech differences to any significant extent. Rather, perturbations of the speech mechanism were most consistent with flaccid dysarthria. As previously proposed in relation to younger patients with IPD ${ }^{4}$ the speech-related dysfunction observed in this cohort is possibly caused by progressive glycogen accumulation in muscles of the velopharynx and/or the various nerves that provide efferent motor innervation for normal execution of function. This study supports previous findings $s^{4,29}$ that suggest that speech-related dysfunction, once it manifests, can persist in the face of continued ERT and targeted clinical interventions such as speech therapy. Dysphagia with aspiration as visualized through videofluoroscopic swallow examination was an additional prevalent finding in our cohort and has clinically significant implications. 
The etiology is possibly similar to that underlying the speechrelated dysfunction, that is, progressive glycogen accumulation in the bulbar musculature and/or the lower motor neurons responsible for its innervation. ${ }^{4}$

The long-term survivors appeared to be functioning at their appropriate respective grade levels at MRA. Moreover, no patient had a prior medical history otherwise suggestive of neurological regression. These findings indicated there was no evidence of gross cognitive impairment or higher-order central nervous system (CNS) dysfunction, although the possibility of subclinical CNS involvement secondary to progressive glycogen accumulation cannot be excluded. Indeed, CNS glycogen accumulation has been documented in autopsy studies of untreated patients with IPD..$^{30}$ Further studies are needed on possible CNS involvement in treated IPD and could, for example, include clinically administered assessment tools and/ or various CNS neuroimaging modalities. Strikingly, all the 10 survivors for whom long-term data were available had either tolerized to ERT or had low anti-rhGAA IgG antibody titers at the time of MRA. We previously described differences in IPDrelated clinical outcomes apparent at various times post-ERT initiation between patients with high sustained antibody titers versus those without. ${ }^{10}$ Findings from this study, in which none of the patients formed high sustained antibody titers, underscore the impact of antibody titers on treatment response. In agreement with recommendations from the manufacturer of alglucosidase alfa (Genzyme), ${ }^{15}$ and elsewhere, ${ }^{32}$ findings from this study also emphasize the importance of monitoring serial anti-rhGAA IgG antibody titers.

Biomarkers that are clinically relevant to IPD, including urinary Glc4 and serum CK, CK-MB, AST, and ALT, were monitored. With the exception of serum CK levels (which remained stable), decreases in these parameters during the first 2 years of therapy were notably correlated with clinical improvement in motor and cardiac function. Glc4 decreased rapidly in response to ERT, suggesting rapid reduction in glycogen upon initiation of therapy. The relative delay in the decrease in CK-MB, AST, and ALT levels may reflect the time required to repair damaged tissues. CK values did not show a significant change between baseline and MRA assessments. After approximately 2 years on therapy, median values for the majority of biomarkers trended upward. This may represent an increase in glycogen storage and muscle damage or an increase in mobility with secondary damage to muscle, and/or may be related to increase in muscle mass with age. It is notable that none of the biomarkers had normalized in the majority of our long-term survivors, correlating with the clinical observations of chronic involvement of skeletal and cardiac muscle in these treated patients.

All the patients in this study met the inclusion criterion of a residual GAA activity in skin fibroblasts and/or muscle biopsy $<1 \%$ of the control mean. All the patients had GAA protein detectable by western blot analysis and were, by definition, CRIM-positive. In contrast, none of the CRIM-negative patients met all the inclusion criteria for this study. In the setting of chronic ERT, it is clear that clinical prognosis is not related solely to a patient's mutation status or CRIM status per se. Indeed, if this were true (which has not been seen in practice), the long-term response to ERT might be expected to be more uniform and predictable. A patient's GAA mutation profile does not appear to operate in isolation but rather in the context of a multitude of various known and yet undiscovered environmental, secondary genetic, and immunologic factors. This is evidenced by the results of various genotype-phenotype studies of Pompe disease, wherein marked variability in clinical outcomes for the same mutation profile has been observed, at least in some instances. ${ }^{33,34}$

Although the CRIM status appears to be prognostic of ERT outcome in the majority of cases, there is evidence that this is not always so. ${ }^{10,11}$ Recently there have been reports of CRIMnegative patients on ERT who have had unexpectedly favorable clinical outcomes, either more akin to those in CRIM-positive patients and/or somewhere in the spectrum between the historically described outcomes for the two categories of CRIM status. $^{35,36}$

The limitations of this study include the paucity of potential study subjects, its retrospective, descriptive, and nonblinded design, and possible selection bias and confirmation bias. To help mitigate the potential influence of confounding factors, analysis was limited to patients with a corrected gestational age of $\leq 6$ months at ERT initiation, who were not in an advanced stage of the disease. The exclusion of patients with survival $<5$ years precluded conclusions from being drawn regarding factors potentially predictive of prolonged survival. For example, in the six patients excluded from this study there may have been factors besides their CRIM-negative status that may have contributed to their comparatively shorter survival. Therefore, the data and conclusions contained herein are not intended as being proof of causation.

The number and percentage of patients with IPD who are predicted to be long-term survivors is expected to increase as ERT continues to make an impact and as further advancements are made in patient management approaches and drug development. Although much has been learned during the past decade, many aspects of the clinical histories of longterm survivors have yet to unfold. Insights regarding the emerging phenotype of the long-term survivor as revealed in our findings are likely to provide a contextual basis for understanding relevant issues. The Pompe Disease Registry, ${ }^{37}$ currently ongoing, is likely to contribute further to the findings of this and other studies. From our study data, it is clear that the constellation of clinical findings for long-term survivors with IPD appears to be in many respects distinct from those associated with late-onset Pompe disease. ${ }^{38,39}$ It will be important to continue to follow these and other long-term survivors with IPD to refine our understanding of factors associated with long-term survival and improved clinical outcomes. An area that warrants further investigation is the possible impact of rhGAA dosage on long-term outcomes. As seen in Supplementary Figure S1 online, 7 
of the 11 long-term survivors in this study had experienced a change in ERT dose at some time point (although, doses remained in the range $20-40 \mathrm{mg} / \mathrm{kg}$ at all time points). This, in conjunction with the discrepant durations of time spent at each dose level, precluded reliable assessment of the potential impact of higher versus standard ERT dose on observed outcomes.

Advancements in the management and treatment of IPD will probably be seen in the coming years; meanwhile, more effective and consistent implementation of current knowledge and approaches is essential.

\section{SUPPLEMENTARY MATERIAL}

Supplementary material is linked to the online version of the paper at http://www.nature.com/gim

\section{ACKNOWLEDGMENTS}

We thank the patients, their families, and the health-care providers who participated in this study. We also thank P. Brian Smith for his assistance related to statistical analysis of CK data. This project was funded partly by the Lysosomal Disease Network, a part of the National Institutes of Health $(\mathrm{NIH})$ Rare Diseases Clinical Research Network, and partly by grant numbers UL1RR024128 and TL1RR024126 (Duke University's Clinical and Translational Science Awards grant) from the National Center for Research Resources (NCRR), a component of the $\mathrm{NIH}$, and the $\mathrm{NIH}$ Roadmap for Medical Research. The contents of this article are solely the responsibility of the authors and do not necessarily represent the official views of the NCRR or the NIH. All data included in this paper were verified and analyzed independently by the authors. P.S.K. had full access to all the data in the study and takes responsibility for the integrity of the data, the accuracy of the data analysis, and the decision to submit the manuscript for publication.

\section{DISCLOSURE}

S.N.P. was funded in part by grant no. TL1RR024126 from the National Institutes of Health (NIH) Roadmap for Medical Research. S.G.B. reports no disclosures. S.P.Y. has received research grant support and honoraria from Genzyme. S.M.D. has received honoraria from Genzyme. E.G.B. is affiliated with research trials at Emory University that include protocols from Genzyme, Protalix Biotherapeutics, Amicus Therapeutics, Shire, and BioMarin Pharmaceutical, all of which potentially fund her salary as a research coordinator. E.M.S. is on the nursing faculty of Centocor Ortho Biotech and receives speaking honoraria related to their product, infliximab (Remicade). She has also received a speaking honorarium from BioMarin Pharmaceutical related to their product for the treatment of mucopolysaccharidosis $\mathrm{VI}$, galsulfase (Naglazyme). L.E.C. has received honoraria from Genzyme; has participated in research supported by Genzyme, PTC Therapeutics, the Leal Foundation, Families of SMA, and Enobia Pharma; has been awarded grant support from the National Skeletal Muscle Research Center; and is a member of the Pompe Registry Board of Advisors for Genzyme. H.N.J. has received research support from the Genzyme.
C.P. reports no disclosures. R.Y.W. has received research funding and speaking honoraria from Shire Human Genetic Therapies unrelated to this study and has had travel and accommodation costs related to attendance of Lysosomal Disease Registry meetings paid for by Genzyme. P.S.K. has received research and grant support as well as honoraria and consulting fees from Genzyme. She is a member of the Pompe Disease and the Gaucher Disease Registry advisory boards for Genzyme and has received research support from grant no. UL1RR024128 from the National Center for Research Resources, a component of the NIH.

\section{REFERENCES}

1. Hirschhorn R, Reuser AJJ. Glycogen storage disease type II: acid a-glucosidase (acid maltase) deficiency. In: Valle D, Scriver CR (eds). Scriver's OMMBID. The Online Metabolic \& Molecular Bases Of Inherited Disease. McGraw-Hill: New York, 2009.

2. Hug G. Pre- and postnatal pathology, enzyme treatment, and unresolved issues in five lysosomal disorders. Pharmacol Rev 1978;30:565-591.

3. Jones HN, Muller CW, Lin M, et al. Oropharyngeal dysphagia in infants and children with infantile Pompe disease. Dysphagia 2010;25:277-283.

4. Muller CS, Jones HN, O'Grady G, Suarez AH, Heller JH, Kishnani PS Language and speech function in children with infantile Pompe disease. $J$ Pediatr Neurol 2009;7(2):147-156.

5. Kishnani PS, Hwu WL, Mandel H, Nicolino M, Yong F, Corzo D; InfantileOnset Pompe Disease Natural History Study Group. A retrospective, multinational, multicenter study on the natural history of infantile-onset Pompe disease. J Pediatr 2006;148:671-676.

6. van den Hout HM, Hop W, van Diggelen OP, et al. The natural course of infantile Pompe's disease: 20 original cases compared with 133 cases from the literature. Pediatrics 2003;112:332-340.

7. Kishnani PS, Corzo D, Nicolino M, et al. Recombinant human acid [alpha]glucosidase: major clinical benefits in infantile-onset Pompe disease. Neurology 2007;68:99-109.

8. Kishnani PS, Corzo D, Leslie ND, et al. Early treatment with alglucosidase alpha prolongs long-term survival of infants with Pompe disease. Pediatr Res 2009;66:329-335.

9. Amalfitano A, Bengur AR, Morse RP, et al. Recombinant human acid alphaglucosidase enzyme therapy for infantile glycogen storage disease type II: results of a phase I/II clinical trial. Genet Med 2001;3:132-138.

10. Banugaria SG, Prater SN, Ng YK, et al. The impact of antibodies on clinical outcomes in diseases treated with therapeutic protein: lessons learned from infantile Pompe disease. Genet Med 2011;13:729-736.

11. Kishnani PS, Goldenberg PC, DeArmey SL, et al. Cross-reactive immunologic material status affects treatment outcomes in Pompe disease infants. Mol Genet Metab 2010;99:26-33.

12. Kishnani PS, Nicolino M, Voit T, et al. Chinese hamster ovary cell-derived recombinant human acid alpha-glucosidase in infantile-onset Pompe disease. J Pediatr 2006;149:89-97.

13. Raben N, Takikita S, Pittis MG, et al. Deconstructing Pompe disease by analyzing single muscle fibers: to see a world in a grain of sand. Autophagy 2007;3:546-552.

14. Vogel M, Staller W, Bühlmeyer K. Left ventricular myocardial mass determined by cross-sectional echocardiography in normal newborns, infants, and children. Pediatr Cardiol 1991;12:143-149.

15. Myozyme [package insert]. Pamphlet. Genzyme Corporation: Cambridge, MA, 2006.

16. Ansong AK, Li JS, Nozik-Grayck E, et al. Electrocardiographic response to enzyme replacement therapy for Pompe disease. Genet Med 2006;8:297-301.

17. Case LE, Kishnani PS. Physical therapy management of Pompe disease. Genet Med 2006;8:318-327.

18. Piper M, Darrah J. Motor Assessment of the Developing Infant. WB Saunders: Philadelphia, PA, 1994.

19. Russell DJ, Rosenbaum PL, Avery LM, Lane M. Gross Motor Function Measure (GMFM - 66 and GMFM - 88) User's Manual. Mac Keith: London, 2002.

20. Case LE, Hanna R, Frush DP, et al. Fractures in children with Pompe disease: a potential long-term complication. Pediatr Radiol 2007;37:437-445. 
21. Kuczmarski RJ, Ogden CL, Grummer-Strawn LM, et al. CDC growth charts: United States. Adv Data 2000;314:1-27.

22. Rollins JD, Collins JS, Holden KR. United States head circumference growth reference charts: birth to 21 years. J Pediatr 2010;156:907-913, 913.e1.

23. Kroos M, Pomponio RJ, van Vliet L, et al.; GAA Database Consortium. Update of the Pompe disease mutation database with 107 sequence variants and a format for severity rating. Hum Mutat 2008;29:E13-E26.

24. Young SP, Zhang $H$, Corzo $D$, et al. Long-term monitoring of patients with infantile-onset Pompe disease on enzyme replacement therapy using a urinary glucose tetrasaccharide biomarker. Genet Med 2009;11:536-541.

25. Wang LY, Ross AK, Li JS, et al. Cardiac arrhythmias following anesthesia induction in infantile-onset Pompe disease: a case series. Paediatr Anaesth 2007;17:738-748.

26. McDowell R, Li JS, Benjamin DK Jr, et al. Arrhythmias in patients receiving enzyme replacement therapy for infantile Pompe disease. Genet Med 2008;10:758-762.

27. Levine JC, Kishnani PS, Chen YT, Herlong JR, Li JS. Cardiac remodeling after enzyme replacement therapy with acid alpha-glucosidase for infants with Pompe disease. Pediatr Cardio/ 2008;29:1033-1042.

28. Chen LR, Chen CA, Chiu SN, et al. Reversal of cardiac dysfunction after enzyme replacement in patients with infantile-onset Pompe disease. J Pediatr 2009;155:271-275.e2.

29. van Gelder CM, van Capelle Cl, Ebbink BJ, et al. Facial-muscle weakness, speech disorders and dysphagia are common in patients with classic infantile Pompe disease treated with enzyme therapy. J Inherit Metab Dis 2011; e-pub ahead of print.

30. Sakurai I, Tosaka A, Mori Y, Imura S, Aoki K. Glycogenosis type II (Pompe). The fourth autopsy case in Japan. Acta Pathol Jpn 1974;24:829-846.
31. Barker PC, Pasquali SK, Darty S, et al. Use of cardiac magnetic resonance imaging to evaluate cardiac structure, function and fibrosis in children with infantile Pompe disease on enzyme replacement therapy. Mol Genet Metab 2010;101:332-337.

32. Kishnani PS, Steiner RD, Bali D, et al.; ACMG Work Group on Management of Pompe Disease. Pompe disease diagnosis and management guideline. Genet Med 2006;8:267-288.

33. Hermans MM, van Leenen D, Kroos MA, et al. Twenty-two novel mutations in the lysosomal alpha-glucosidase gene (GAA) underscore the genotypephenotype correlation in glycogen storage disease type II. Hum Mutat 2004;23:47-56.

34. Kroos MA, Kirschner J, Gellerich FN, et al. A case of childhood Pompe disease demonstrating phenotypic variability of p.Asp645Asn. Neuromuscul Disord 2004;14:371-374.

35. Rohrbach $M$, Klein A, Köhli-Wiesner A, et al. CRIM-negative infantile Pompe disease: 42-month treatment outcome. J Inherit Metab Dis 2010;33:751-757.

36. Abbott MA, Prater SN, Banugaria SG, et al. Atypical immunologic response in a patient with CRIM-negative Pompe disease. Mol Genet Metab 2011;104:583-586.

37. Byrne BJ, Kishnani PS, Case LE, et al. Pompe disease: design, methodology, and early findings from the Pompe Registry. Mol Genet Metab 2011;103:1-11.

38. American Association of Neuromuscular \& Electrodiagnostic Medicine. Diagnostic criteria for late-onset (childhood and adult) Pompe disease. Muscle Nerve 2009;40(1):149-160.

39. Muller-Felber W, Horvath R, Gempel K, et al. Late onset Pompe disease: clinical and neurophysiological spectrum of 38 patients including long- term follow-up in 18 patients. Neuromuscul Disord 2007;7(9-10):698-706. 